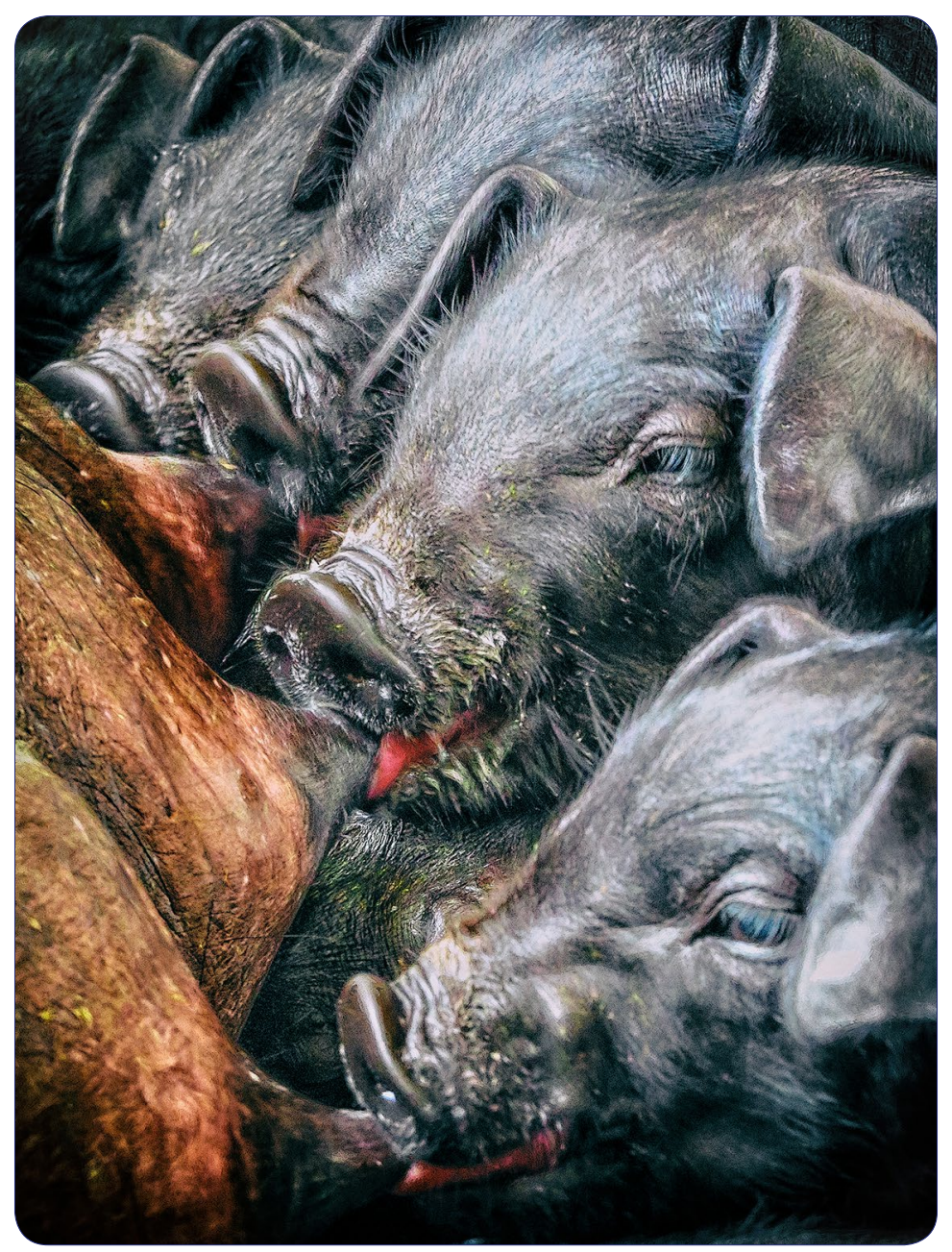

\title{
Genomic inbreeding coefficients based on the distribution of the length of runs of homozygosity in a closed line of Iberian pigs
}

Gomez-Raya et al. 


\title{
Genomic inbreeding coefficients based on the distribution of the length of runs of homozygosity in a closed line of Iberian pigs
}

\author{
Luis Gomez-Raya*, Carmen Rodríguez, Carmen Barragán and Luis Silió
}

\begin{abstract}
Background: The increasing availability of DNA markers provides new metrics of inbreeding based on single nucleotide polymorphisms (SNPs), i.e. molecular inbreeding or the proportion of runs of homozygosity (ROH), as alternatives to traditional pedigree-based inbreeding coefficients. However, none of these metrics incorporate the length of $\mathrm{ROH}$ as an indicator of recent inbreeding. Novel inbreeding coefficients that incorporate length of $\mathrm{ROH}$ as a random variable with an associated density are investigated.
\end{abstract}

Methods: New inbreeding metrics based on the distribution of the length of $\mathrm{ROH}$ are proposed: (1) the Kolmolgorov-Smirnov test, (2) a function of the quantiles of the cumulative distribution function of an individual versus the population, and (3) fitting of an exponential distribution to $\mathrm{ROH}$ lengths (mean, variance, and the probability of drawing at random a ROH larger than a given threshold). The new inbreeding and pedigree-based metrics were compared using 217 sows of an Iberian line that belong to three groups: C1 (conservation), C2 (conservation derived from C1), and S (selected and derived from C1), with complete pedigrees and genotyped for 35,023 SNPS.

Results: Correlations between pedigree-based and the new genomic inbreeding coefficients ranged from 0.22 to 0.72 but most ranged from 0.60 to 0.70 . The correlation between quantile chromosomal inbreeding coefficients (using molecular information of just one chromosome at the time) and chromosomal length was $0.84(\mathrm{SE}=0.14)$, supporting the hypothesis that these coefficients incorporate information on $\mathrm{ROH}$ length as an indication of recent inbreeding. Kolmogorov-Smirnov and exponential chromosomal inbreeding coefficients were also correlated with chromosomal length (0.57). Chromosome 1 had the largest quantile $\mathrm{ROH}$ inbreeding coefficient (largest $\mathrm{ROH}$ sizes), whereas chromosome 10 had the lowest (shortest $\mathrm{ROH}$ sizes). Selection for lean growth increased $\mathrm{ROH}$-based inbreeding coefficients for group $S$ when compared to unselected groups C1 and C2. At the chromosomal level, this comparison showed that the level of autozygosity and the length of $\mathrm{ROH}$ for most of the autosomes increased in the selection line.

Conclusions: Quantile and exponential probability inbreeding coefficients using ROH length as a random variable provide additional information about recent inbreeding compared to existing inbreeding coefficients such as molecular, pedigree-based or total $\mathrm{ROH}$ content inbreeding coefficients.

\section{Background}

The inbreeding coefficient of an individual is the probability that two alleles at a locus in that individual are identical by descent [1]. The inbreeding coefficient is a key

\footnotetext{
*Correspondence: gomez.luis@inia.es

Departamento de Mejora Genética Animal, Instituto Nacional de

Investigación y Tecnología Agraria y Alimentaria (INIA), Ctra. de La Coruña km 7, 28040 Madrid, Spain
}

parameter to understand the amount of matings between related individuals that have taken place in a population. Inbreeding leads to an increase in homozygosity, which, in turn, reduces performance of production traits (inbreeding depression), reduces fitness and compromises long-term viability of the population $[2,3]$. Therefore, control of inbreeding is itself an objective in animal production or conservation genetics [4]. 
In farm animals, coefficients of inbreeding are systematically computed from pedigree records using path coefficients [5]. If pedigrees are not available, inbreeding coefficients can be calculated using molecular information. In particular, genome-wide single nucleotide polymorphism (SNP) bead chips are used to assess levels of homozygosity [6] or to estimate pedigree-based inbreeding coefficients $[7,8]$. These approaches assume that SNPs are unlinked and they do not make use of all available information. However, SNPs are physically linked and alleles at linked markers on the same homologous chromosome are inherited together unless a recombination event occurs between them.

Runs of homozygosity (ROH) are defined as continuous and uninterrupted stretches of DNA sequences without heterozygosity in diploid state [9]. Presence of long $\mathrm{ROH}$ can imply recent inbreeding, which can be used to estimate genome-wide autozygosity and inbreeding coefficients, as suggested by Keller et al. [10]. ROH has been used to investigate inbreeding in human [11-13], cattle $[14,15]$, and pig populations $[16,17]$.

The generation of $\mathrm{ROH}$ is explained in Fig. 1. "A" represents a common ancestor of parents " $D$ " and "E" of individual "F". Individual " $F$ " has a ROH fragment identical by descent, which is delimited by blue arrows. The line under "A" represents one of the two homologous chromosomes (in blue), which will generate the $\mathrm{ROH}$ in individual " $\mathrm{F}$ ". Colors other than blue are used to represent chromosomes of different origin. For this example, there were recombination events in paths "A" to "B", "B" to " $D$ ", and " $C$ " to " $E$ "; there were no recombination events in paths "A" to "C", "D" to "F" or " $E$ " to "F". The effect of recombination is to break down the length of the homologous chromosome in steps from ancestor "A" to individual "F".

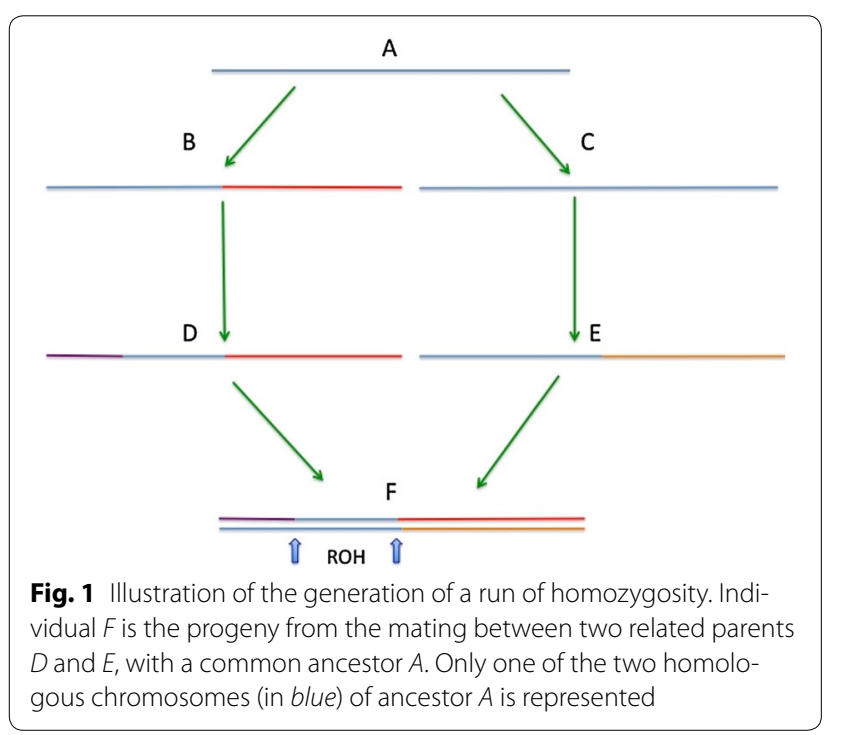

This illustration can be used to identify the main factors that affect the length of ROH: (a) the number of steps in the paths from "A" to " $\mathrm{F}$ " (opportunities for recombination, green arrows in the scheme), where a small number of steps (recent inbreeding) results in longer $\mathrm{ROH}$; (b) the recombination rate in a chromosome (which can vary at the population or individual level); and (c) the length of the chromosome, with longer chromosomes yielding longer $\mathrm{ROH}$ because the longer the chromosome is in the ancestor, the longer is the $\mathrm{ROH}$ fragment in the individual in which inbreeding is assessed. However, the latter has not been proven empirically.

The approach used to compute inbreeding coefficients based on $\mathrm{ROH}$ requires calculating the total length of $\mathrm{ROH}$ covering the genome of an individual (for a given minimum number of contiguous homozygous SNPs) divided by the length of the genome [11, 18]. As stated above, recent inbreeding is associated to larger $\mathrm{ROH}$ fragments [10-19]. However, it is not well established either how to make a comparison between individuals with different numbers and lengths of $\mathrm{ROH}$ fragments or how to use the length of $\mathrm{ROH}$ to estimate recent inbreeding.

The objective of this paper was to investigate the use of $\mathrm{ROH}$ length as a random variable with an associated distribution or probability density to derive new inbreeding coefficients: (1) a method based on the Kolmogorov-Smirnov test, (2) a method based on quantiles of the distribution of the length of $\mathrm{ROH}$, and (3) a method based on fitting an exponential distribution to the $\mathrm{ROH}-$ length distribution. These inbreeding coefficients were compared to SNP-based homozygosity metrics and pedigree inbreeding coefficients. It is shown that the new coefficients provide additional information on recent inbreeding. The new inbreeding coefficients were used to investigate inbreeding in a closed line of Iberian pigs maintained in a conservation program and to investigate the effect of selection on inbreeding.

\section{Methods}

\section{Torbiscal line pedigree}

The pigs of this study belong to a composite line (Torbiscal) resulting from the blending of four ancient Spanish and Portuguese strains of Iberian breeds in 1944 that was genetically isolated from 1963 to 2013 [20]. A complete genealogy of 4524 entries (individual-sire-dam) was available. The inbreeding coefficient $\left(F_{p e d}\right)$ and the number of discrete generation equivalents $(E q G)[21]$ were calculated based on this pedigree for each individual with respect to a base generation of unknown parents [22]. A partition of individual inbreeding coefficients into two components, new $\left(F_{\text {ped-new }}\right)$ and old $\left(F_{\text {ped-old }}\right)$, was performed using the expressions proposed by Hinrichs et al. [23]: 


$$
F_{i, o l d}(u, t)=\left[F_{i}(0, t)-F_{i}(u, t)\right] /\left[1-F_{i}(u, t)\right]
$$

and

$$
F_{i, \text { new }}(u, t)=F_{i}(0, t)-F_{i, \text { old }}(u, t) \text { for } 0<u<t,
$$

where 0 is the base generation, $u$ is any intermediate generation, and $t$ is the generation of the $i$-th individual. The intermediate generation $(u)$ constituted a base by assuming that parents of pigs born in 1980 were unknown. The comparison between different pedigree and genomic inbreeding metrics was performed on data consisting of 217 sows from three related cohorts: 54 sows born between 1994 and 1998 (C1 group) with an average number of discrete generation equivalents $E q G_{C 1}=21.04$ $(\mathrm{SD}=0.57)$ and an average pedigree inbreeding coefficient $F_{C 1}=0.15(\mathrm{SD}=0.01)$, a group of 54 sows (S) contemporary to the $\mathrm{C} 1$ group coming from a sub-line experimentally selected for lean growth along seven generations with $E q G_{S}=22.72(\mathrm{SD}=0.78)$ and $F_{S}=0.21$ ( $\mathrm{SD}=0.019)$, and a third group of 109 sows $(\mathrm{C} 2)$ descendent from the $\mathrm{C} 1$ group that were born between 2004 and 2010 and with $E q G_{C 2}=26.13(\mathrm{SD}=0.74)$ and $F_{C 2}=0.18$ $(\mathrm{SD}=0.02)$. Details of the selection experiment based on records of backfat thickness and growth can be found in Rodriguez et al. [24].

\section{Genotyping and SNP-based metrics of inbreeding}

DNA was isolated from blood using a standard phenol/ chloroform protocol and genotyped with the Illumina Porcine SNP60 BeadChip [25] and the Infinium HD Assay Ultra protocol (Illumina Inc.). Genotypes of 62,163 SNPs were called with the GenomeStudio software (Illumina). In addition, DNA from 17 Iberian pigs representing the main breeding nuclei of this breed were analyzed to identify SNPs of good quality that were monomorphic or had very low minor allele frequency (MAF) in the Torbiscal line. Quality control of genotypes was performed according to the following criteria: call rate for the individual $>0.96$; SNPs with a call rate $>0.99$; GenTrain score (measure of the reliability of the SNP detection based on the distribution of genotypic classes) $>0.70$; $A B R$ mean (mean of the normalized intensity of the heterozygote cluster) $>0.35$; and MAF $>0.05$. SNPs located on sex chromosomes, those not mapped in the Sscrofa10.2 assembly (http://gbi.agrsci.dk/pig/sscrofa10_2_annotation/), or those with inconsistent inheritance from dam to daughter were also removed. Based on these criteria 35,023 SNPs were retained and used for further analyses.

Genomic inbreeding coefficients based on the distribution of the length of ROH

A minimum number of contiguous SNPs with homozygous genotypes are required for declaring a stretch of
DNA as a $\mathrm{ROH}$ in an individual because short tracts of homozygosity are rather common due to strong linkage disequilibrium. $\mathrm{ROH}$ length can be expressed either as the number of contiguous homozygous SNPs, or as the length measured in units of physical distance in $\mathrm{Mb}$. These two measures of $\mathrm{ROH}$ length are highly correlated and both represent estimates of autozygosity (two chromosomal segments inherited from each parent that are identical from a common ancestor) since only a limited number of SNPs are genotyped within a DNA segment. Because of the exploratory nature of this paper, several alternative minimum numbers of contiguous SNPs (5, 15,25 , and 35 ) were used to define a $\mathrm{ROH}$ in order to investigate their impact on the novel inbreeding coefficients based either on the length of $\mathrm{ROH}$ estimated as the number of contiguous homozygous SNPs or in physical distance $(\mathrm{Mb})$. For the majority of the methods, estimates of individual autozygosity (I-ROH) were taken as a deviation from a reference population or group (A-ROH). Unless stated otherwise, the reference population will consist of all individuals with available genotypes. Source code in R language (http://www.r-project.org/) for estimating the inbreeding coefficients and a small example for two individuals are provided as supplementary material in Additional files 1, 2, 3, 4 and 5.

\section{KS-ROH inbreeding coefficient}

The Kolmogorov-Smirnov test (KS test) is a non-parametric test to compare two one-dimensional probability distributions. The Kolmogorov-Smirnov statistic $(D)$ quantifies the distance between a given cumulative distribution $(\mathrm{T})$ and the cumulative distribution of a reference distribution (S) and is computed as:

$$
D=\max _{x}\left|F_{T}(x)-F_{S}(x)\right|,
$$

where $F_{T}(x)$ and $F_{S}(x)$ are the empirical cumulative distributions of $\mathrm{T}$ and $\mathrm{S}$ at point $x$, respectively. Therefore, $D$ measures the largest distance between the two cumulative distribution functions. We used a modification of the KS test to compute the inbreeding coefficient of an individual based on the length of $\mathrm{ROH}$ by computing the KS statistic of the distribution of the lengths of $\mathrm{ROH}$ of the individual compared to a reference distribution that consists of the lengths of all $\mathrm{ROH}$ of all individuals (e.g., population, strain). Thus, the KS inbreeding coefficient measures the maximum distance between two cumulative distribution functions $(\mathrm{CDF})$ :

$$
F_{R O H-K S}=\max _{x}\left(F_{I-R O H}(x)-F_{A-R O H}(x)\right),
$$

where $F_{I-R O H}(x)$ and $F_{A-R O H}(x)$ are the cumulative distributions at point $\mathrm{x}$ that has the maximum distance between the two distributions. The subscripts I-ROH and $\mathrm{A}-\mathrm{ROH}$ refer to individual and all individuals (or 
reference population), respectively. Note that the absolute value was dropped from this test to allow for positive or negative deviations from the reference population.

\section{Quantile-ROH inbreeding coefficient}

Quantiles are points taken at regular intervals from the $\mathrm{CDF}$ of a random variable. The $\mathrm{ROH}-\mathrm{Q}$ inbreeding coefficient of an individual is defined as the sum of the differences between the quantiles of the CDF of ROH lengths of the individual $\left(q_{I-R O H}\right)$ and the quantiles of the reference population $\left(q_{A-R O H}\right)$ :

$$
F_{R O H-Q}=\sum_{i=1}^{n q} \frac{q_{I-R O H}-q_{A-R O H}}{\sqrt{2}},
$$

where $n q$ is the number of quantiles, $q_{I-R O H}$ and $q_{A-R O H}$ are the quantiles of the CDF of $\mathrm{ROH}$ length of the individual and of a reference population. This equation can be obtained by considering points in a Q-Q plot with coordinates $q_{\mathrm{I}-\mathrm{ROH}}$ and $q_{\mathrm{A}-\mathrm{ROH}}$, and measuring their distance to the diagonal (which is the expected distribution if both CDF would be equal; i.e. $f(x)=x$ ). The sum of all distances is $F_{R O H-Q}$. Note that the distance between each point coordinate and the diagonal line $(f(x)=x)$ can be positive or negative. Percentiles were the quantiles used in this study.

\section{Exponential-ROH inbreeding coefficient}

Following Clark [26], the distribution of the length of an autozygous segment is expected to follow an exponential distribution. After fitting an exponential distribution to the distribution of the $\mathrm{ROH}$ lengths of an individual and of all individuals (reference population), the coefficient of inbreeding of the individual can be estimated based on the following statistics:

(a) Using the mean of the exponential density:

$$
F_{R O H-E^{m}}=\frac{1}{\lambda_{I-R O H}}-\frac{1}{\lambda_{A-R O H}},
$$

where $\lambda_{I-R O H}$ and $\lambda_{A-R O H}$ are the rates of the fitted exponential distribution for the individual and for all individuals, respectively.

(b) Using the variance of the exponential density:

$$
F_{R O H-E^{v}}=\frac{1}{\lambda_{I-R O H}^{2}}-\frac{1}{\lambda_{A-R O H}^{2}} .
$$

(c) Using the integral of the fitted exponential density for the individual from a threshold $\mathrm{T}$ to $\infty$ to calculate the inbreeding coefficient of the individual based on the probability of getting an $\mathrm{ROH}$ fragment with length larger than $\mathrm{T}$ as:

$$
F_{R O H-E^{p}}=\int_{T}^{\infty} \lambda_{I-R O H} e^{-\left(\lambda_{I-R O H}\right) x} d(x),
$$

where $\mathrm{T}$ is the threshold and $\mathrm{x}$ the length of the $\mathrm{ROH}$. This genomic inbreeding coefficient is an estimate of the degree of autozygosity of an individual, and being a probability, it is forced to range from 0 to 1 . The threshold is arbitrary but comparison between individuals is feasible when the same threshold is used for individuals from the same population typed with the same array.

Note that in the above equations, terms that apply to all individuals (i.e. the exponential distribution with rate $\left.\lambda_{A-R O H}\right)$ is the same for all individuals and, therefore, does not affect the ranking of individuals based on their inbreeding coefficients. Results will be provided for all three coefficients comparing correlations of these coefficients with traditional inbreeding coefficients. However, only $F_{R O H-E^{m}}$ or $F_{R O H-E^{p}}$ coefficients will be discussed in other sections of the paper in order to reduce the number of tables and figures.

\section{Traditional inbreeding coefficients}

Correlations were estimated between the new inbreeding coefficients based on the length of $\mathrm{ROH}$ and the following traditional inbreeding coefficients: (1) pedigree-based inbreeding coefficient $\left(F_{\text {ped }}\right)$ computed for each individual by tracing the pedigree back to the founder animals; (2) pedigree-based new inbreeding coefficient $\left(F_{\text {ped-new }}\right)$ based on the equations proposed by Hinrichs et al. [23] with breeding animals born in 1980 as the intermediate base generation; (3) pedigree-based old inbreeding coefficient $\left(F_{\text {ped-old }}\right)$ based on the equations proposed by Hinrichs et al. [23] after ignoring all inbreeding generated from 1980 on; (4) molecular inbreeding coefficient $\left(F_{\text {Mol }}\right)$, defined as the proportion of genotyped SNPs at which an individual is homozygous (identical by state) [6]; and (5) total $\mathrm{ROH}$ content based metric of homozygosity [11] calculated for the autosomal genome as $F_{R O H}=L_{R O H} / L_{A U T O}$, where $L_{R O H}$ is the total $\mathrm{ROH}$ length of the individual and $L_{A U T O}$ is the length of the autosomal genome [11]. Identification of $\mathrm{ROH}$ was performed with the program PLINK (http://pngu.mgh.harvard.edu/purcell/plink/). In order to adapt to the much lower density of SNPs than those used by McQuillan [11], the conditions for declaring a $\mathrm{ROH}$ included a sliding window of 15 SNPs, allowing two missing calls and one heterozygous SNP per window; a $\mathrm{ROH}$ was declared if it had a length of at least $100 \mathrm{~kb}$ and contained 15 or 
more SNPs. The minimum required density was one SNP per $500 \mathrm{~kb}$ and the maximum gap allowed between any two consecutive SNPs was $1000 \mathrm{~kb}$. Other options were according to the default settings in the program.

\section{Results}

Figure 2 shows the distributional properties of two extreme sows with pedigree inbreeding coefficients of 0.13 (sow 13304804) and 0.38 (sow 18705308) to illustrate the statistical principles of the newly developed metrics. The probability densities of $\mathrm{ROH}$ lengths for the two sows were clearly different, with sow 18705308 having longer $\mathrm{ROH}$ fragments. This is also shown when plotting the cumulative distribution functions of $\mathrm{ROH}$ lengths for the two sows. The Kolmogorov-Smirnov method measures the maximum distance between two cumulative distributions and the observed differences in the cumulative distribution for the $\mathrm{ROH}$ lengths of these two individuals justify this metric. In the same manner, the two Q-Q plots for these two sows, which are graphical representations of the quantiles of each sow versus the quantiles of all sows in the dataset, show very large differences in the distributions for these two sows. The proposed quantile inbreeding coefficient is calculated by summing up the distances between the quantiles of the distribution of $\mathrm{ROH}$ lengths for a sow (points in the graph) and the diagonal. The shape of the line that represents the $Q-Q$ plots, i.e. whether it is curved or linear, may exemplify
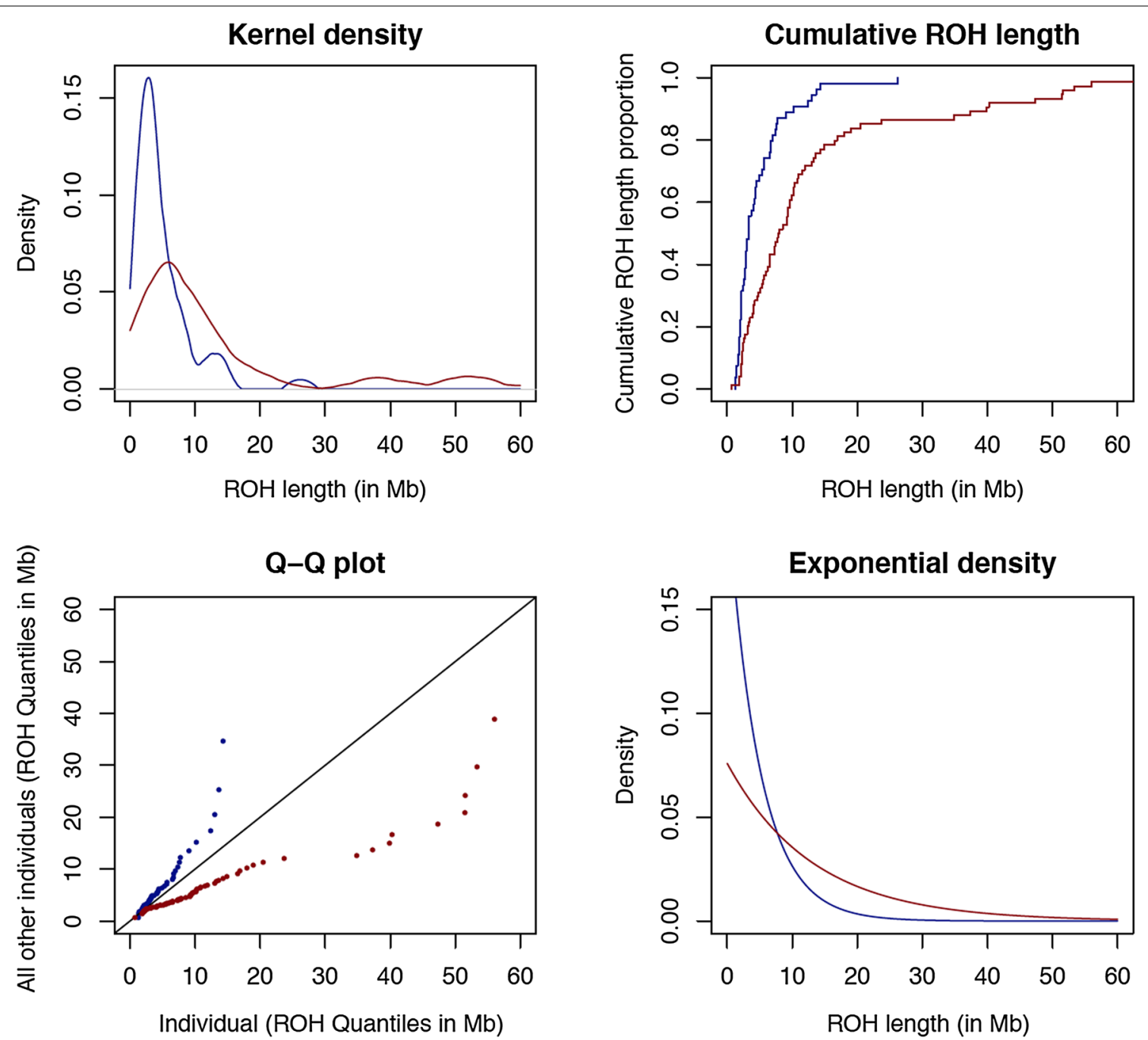

Fig. 2 Distributional properties of the distribution of the length of runs of homozygosity (ROH). Kernel density, Q-Q plots, cumulative ROH length, and adjusted exponential density for the distributions of the length of $\mathrm{ROH}$ of two individuals with extreme pedigree inbreeding coefficients: sow 18705308 (highest inbreeding; dark red) and sow 13304804 (lowest inbreeding; dark blue). Minimum number of SNPs to declare a ROH $>35, \mathrm{ROH}$ measured in $\mathrm{Mb}$ 
episodes of recent or old inbreeding. Finally, the adjusted exponential densities of the two sows show differences in the rate at which the highly inbred sow has longer $\mathrm{ROH}$ fragments. The right hand side of this graph is similar to the observed kernel density, whereas the distribution to the left of the peak does not seem to fit an exponential density. In summary, there are differences in the distribution of $\mathrm{ROH}$ lengths between individuals depending on their inbreeding history.

Figure 3 shows the histograms of pedigree and $\mathrm{ROH}-$ based inbreeding coefficients to better picture the distribution of the novel inbreeding coefficients. The distributions of both $F_{p e d}$ and $F_{M o l}$, with respective means of $0.18(\mathrm{SD}=0.03)$ and 0.74 $(\mathrm{SD}=0.02)$, are similar but have a different range of values. All ROH-based coefficients, except $F_{R O H-E}^{p}$, have values that fall outside the range of $0-1$, which is problematic when comparing inbreeding coefficients between populations or species. Both traditional and novel inbreeding coefficients were able to identify the sow with the highest inbreeding (18705308), i.e., the most extreme value on the right hand side of all histograms.

In order to illustrate the new metrics, correlations between traditional and $\mathrm{ROH}$-based inbreeding coefficients are in Tables 1 and 2 when considering length

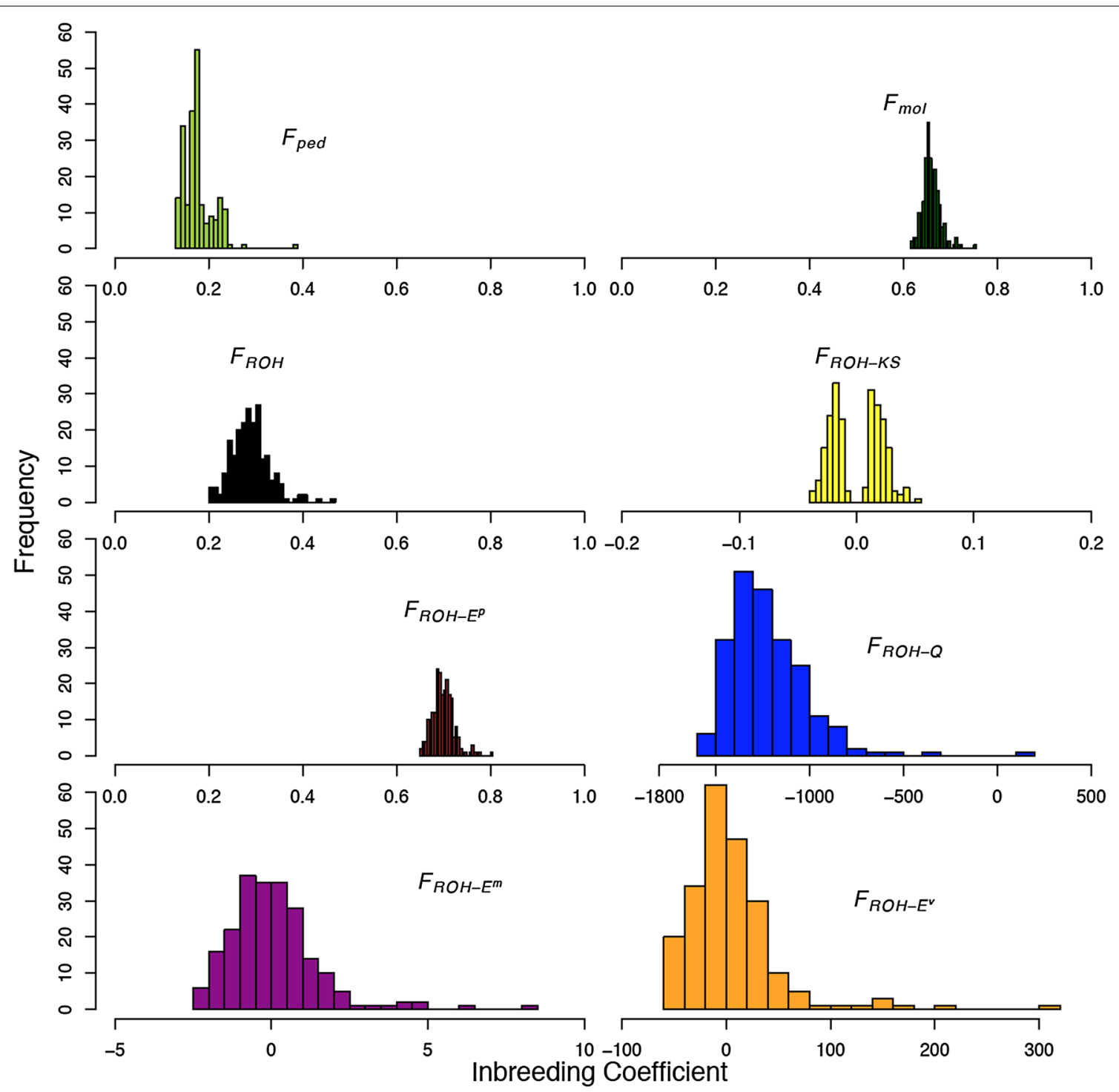

Fig. 3 Histograms of frequencies of pedigree and genomic inbreeding coefficients. The histograms are based on ROH lengths from 217 Torbiscal Iberian sows (minimum number of SNPs to declare a $\mathrm{ROH}>5$ for quantile and exponential, $\mathrm{ROH}$-length in $\mathrm{Mb}$ ). The threshold for exponential- $p$ was $5 \mathrm{Mb}$. Abbreviations for inbreeding coefficients: $F_{\text {ped' }}$ pedigree; $F_{\text {mol' }}$ molecular; $F_{R O H}$, total ROH content; $F_{R O H-K S}$, Kolmolgorov-Smirnov; $F_{R O H-E}^{p}$ probability exponential; $F_{R O H-Q^{\prime}}$ quantile; $F_{R O H-E^{\prime}}^{m}$ mean exponential; $F_{R O H-E^{\prime}}^{V}$ variance exponential 
Table 1 Correlations between current and new genomic inbreeding coefficients measured in number of SNPs and using alternative minimum numbers of SNPs when declaring a ROH

\begin{tabular}{|c|c|c|c|c|c|}
\hline & $F_{\text {ROH-KS }}$ & $F_{\text {ROH-Q }}$ & $F_{R O H-E}^{m}$ & $F_{R O H-E}^{v}$ & $F_{R O H-E}^{p}$ \\
\hline \multicolumn{6}{|c|}{ Minimum number of SNPs $>5$} \\
\hline$F_{p e d}$ & 0.223 & 0.548 & 0.716 & 0.723 & 0.690 \\
\hline$F_{\text {ped-new }}$ & 0.226 & 0.548 & 0.717 & 0.724 & 0.691 \\
\hline$F_{\text {ped-old }}$ & -0.056 & 0.170 & 0.171 & 0.164 & 0.174 \\
\hline$F_{\text {Mol }}$ & 0.406 & 0.749 & 0.963 & 0.945 & 0.969 \\
\hline$F_{R O H}$ & 0.407 & 0.749 & 0.948 & 0.927 & 0.960 \\
\hline \multicolumn{6}{|c|}{ Minimum number of SNPs $>15$} \\
\hline$F_{p e d}$ & 0.472 & 0.663 & 0.672 & 0.677 & 0.630 \\
\hline$F_{\text {ped-new }}$ & 0.473 & 0.663 & 0.672 & 0.677 & 0.630 \\
\hline$F_{\text {ped-old }}$ & 0.081 & 0.190 & 0.187 & 0.183 & 0.176 \\
\hline$F_{\text {Mol }}$ & 0.675 & 0.892 & 0.902 & 0.885 & 0.892 \\
\hline$F_{R O H}$ & 0.897 & 0.877 & 0.884 & 0.870 & 0.877 \\
\hline \multicolumn{6}{|c|}{ Minimum number of SNPs $>25$} \\
\hline$F_{p e d}$ & 0.500 & 0.655 & 0.651 & 0.667 & 0.587 \\
\hline$F_{\text {ped-new }}$ & 0.501 & 0.655 & 0.651 & 0.668 & 0.587 \\
\hline$F_{\text {ped-old }}$ & 0.113 & 0.199 & 0.199 & 0.188 & 0.199 \\
\hline$F_{\mathrm{MOl}}$ & 0.688 & 0.866 & 0.857 & 0.850 & 0.830 \\
\hline$F_{\mathrm{ROH}}$ & 0.676 & 0.837 & 0.828 & 0.818 & 0.802 \\
\hline \multicolumn{6}{|c|}{ Minimum number of SNPs $>35$} \\
\hline$F_{p e d}$ & 0.470 & 0.631 & 0.621 & 0.650 & 0.544 \\
\hline$F_{\text {ped-new }}$ & 0.470 & 0.630 & 0.620 & 0.650 & 0.543 \\
\hline$F_{\text {ped-old }}$ & 0.167 & 0.224 & 0.225 & 0.212 & 0.231 \\
\hline$F_{\mathrm{Mol}}$ & 0.655 & 0.829 & 0.813 & 0.815 & 0.774 \\
\hline$F_{R O H}$ & 0.638 & 0.796 & 0.780 & 0.780 & 0.744 \\
\hline
\end{tabular}

Current inbreeding coefficients are pedigree $\left(F_{\text {ped }} F_{\text {ped-new }}\right.$ and $\left.F_{\text {ped-old }}\right)$, molecular $\left(F_{M o}\right)$ and total $\mathrm{ROH}$ content $\mathrm{ROH}\left(F_{\mathrm{ROH}}\right)$; novel genomic inbreeding coefficients are Kolmolgorov-Smirnov $\left(F_{\mathrm{ROH}-\mathrm{KS}}\right)$, quantile $\left(F_{\mathrm{ROH}-\mathrm{Q}}\right)$, exponential mean, variance and probability $\left(F_{R O H-E^{\prime}}^{m} F_{R O H-E_{1}}^{v} F_{R O H-E}^{p}\right)$; standard errors range from 0.017 to 0.068
Table 2 Correlations between current and new genomic inbreeding coefficients measured in length of ROH in $\mathrm{Mb}$ and using alternative minimum numbers of SNPs when declaring a ROH

\begin{tabular}{|c|c|c|c|c|c|}
\hline & $F_{\text {ROH-КS }}$ & $F_{\mathrm{ROH}-\mathrm{Q}}$ & $F_{R O H-E}^{m}$ & $F_{R O H-E}^{v}$ & $F_{R O H-E}^{p}$ \\
\hline \multicolumn{6}{|c|}{ Minimum number of SNPs $>5$} \\
\hline$F_{\text {ped }}$ & 0.166 & 0.510 & 0.701 & 0.713 & 0.704 \\
\hline$F_{\text {ped-new }}$ & 0.169 & 0.510 & 0.707 & 0.714 & 0.705 \\
\hline$F_{\text {ped-old }}$ & -0.081 & 0.150 & 0.158 & 0.151 & 0.161 \\
\hline$F_{\mathrm{Mol}}$ & 0.317 & 0.696 & 0.960 & 0.939 & 0.960 \\
\hline$F_{\mathrm{ROH}}$ & 0.295 & 0.717 & 0.956 & 0.931 & 0.956 \\
\hline \multicolumn{6}{|c|}{ Minimum number of SNPs $>15$} \\
\hline$F_{p e d}$ & 0.447 & 0.645 & 0.652 & 0.656 & 0.625 \\
\hline$F_{\text {ped-new }}$ & 0.447 & 0.645 & 0.653 & 0.657 & 0.625 \\
\hline$F_{\text {ped-old }}$ & 0.110 & 0.176 & 0.176 & 0.171 & 0.172 \\
\hline$F_{\mathrm{Mol}}$ & 0.613 & 0.878 & 0.894 & 0.875 & 0.890 \\
\hline$F_{R O H}$ & 0.897 & 0.883 & 0.893 & 0.865 & 0.893 \\
\hline \multicolumn{6}{|c|}{ Minimum number of SNPs $>25$} \\
\hline$F_{p e d}$ & 0.459 & 0.618 & 0.614 & 0.631 & 0.561 \\
\hline$F_{\text {ped-new }}$ & 0.458 & 0.619 & 0.614 & 0.632 & 0.560 \\
\hline$F_{\text {ped-old }}$ & 0.168 & 0.184 & 0.185 & 0.171 & 0.194 \\
\hline$F_{\mathrm{Mol}}$ & 0.587 & 0.850 & 0.840 & 0.835 & 0.813 \\
\hline$F_{R O H}$ & 0.580 & 0.843 & 0.832 & 0.823 & 0.808 \\
\hline \multicolumn{6}{|c|}{ Minimum number of SNPs $>35$} \\
\hline$F_{p e d}$ & 0.445 & 0.586 & 0.576 & 0.607 & 0.507 \\
\hline$F_{\text {ped-new }}$ & 0.443 & 0.585 & 0.576 & 0.607 & 0.506 \\
\hline$F_{\text {ped-old }}$ & 0.244 & 0.197 & 0.198 & 0.182 & 0.140 \\
\hline$F_{\text {Mol }}$ & 0.607 & 0.809 & 0.792 & 0.797 & 0.675 \\
\hline$F_{R O H}$ & 0.599 & 0.801 & 0.794 & 0.786 & 0.747 \\
\hline
\end{tabular}

Current inbreeding coefficients are pedigree $\left(F_{\text {ped, }}, F_{\text {ped-new }}\right.$ and $\left.F_{\text {ped-old }}\right)$, molecular $\left(F_{M o l}\right)$ and total ROH content $\left(F_{R O H}\right)$; novel genomic inbreeding coefficients are Kolmolgorov-Smirnov $\left(F_{\mathrm{ROH}-\mathrm{KS}}\right)$, quantile $\left(F_{\mathrm{ROH}-\mathrm{Q}}\right)$ and exponential mean, variance and probability $\left(F_{R O H-E}^{m} F_{R O H-E}^{v} F_{R O H-E}^{p}\right)$; standard errors range from 0.015 to 0.068 of $\mathrm{ROH}$ either as the number of SNPs (Table 1) or as physical distance in $\mathrm{Mb}$ (Table 2). Correlations were computed for a range of minimum number of SNPs $(5,15,25$, and 35$)$ in a DNA stretch tested for autozygosity. The correlation between $\mathrm{ROH}$-based and pedigree $\left(F_{\text {ped }}\right)$ inbreeding coefficients ranged from 0.22 to 0.72 (Table 1 ) and from 0.17 to 0.71 (Table 2). All correlations were lower when $\mathrm{ROH}$ length was measured in $\mathrm{Mb}$. Partitioning individual inbreeding coefficients into new $\left(F_{\text {ped-new }}\right)$ and old $\left(F_{\text {ped-old }}\right)$ inbreeding, allowed the proposed $\mathrm{ROH}$ metrics to be correlated with recent and past inbreeding. For all $\mathrm{ROH}$ inbreeding coefficients, regardless of the minimum number of SNPs used and whether $\mathrm{ROH}$ length was based on the number of SNPs or physical distance, the correlations of the novel $\mathrm{ROH}$ metrics with $F_{\text {ped-new }}$ were very similar to their correlations with $F_{\text {ped }}$, whereas the correlation with $F_{\text {ped-old }}$ was small. Except for $F_{R O H-K S}$ (minimum number of SNPs
$>5$ ), the novel metrics were also highly correlated with $F_{M o l}$ and $F_{R O H}$, as expected. Correlations of the parametric inbreeding coefficients $F_{R O H-E}^{m}, F_{R O H-E}^{v}$ and $F_{R O H-E}^{p}$ with $F_{p e d}, F_{M o l}$ and $F_{R O H}$ were higher when the minimum number of SNPs to determine $\mathrm{ROH}$ was equal to five, which is as expected since the use of a larger number of SNPs truncates the parametric distribution. In contrast, correlations of inbreeding coefficients based on the nonparametric distributions $F_{R O H-K S}$ and $F_{R O H-Q}$, with $F_{p e d}$, $F_{M o l}$ and $F_{R O H}$ were higher for an intermediate minimum number of SNPs. For example, the KS inbreeding coefficient gave a low correlation with pedigree inbreeding coefficients of $0.22,0.47,0.50$ and 0.47 for minimum number of SNPs equal to $5,15,25$, and 35 , respectively (Table 1). This method uses the largest distance between the two cumulative distributions, which may be better estimated by ignoring small $\mathrm{ROH}$ fragments. For $\mathrm{ROH}$ with a minimum number of SNPs greater than 5 , 
correlations between $F_{M o l}$ and inbreeding coefficients based on the length of $\mathrm{ROH}$ ranged from 0.41 to 0.97 (Table 1), and from 0.32 to 0.96 (Table 2). Similarly, total $\mathrm{ROH}$ content was highly correlated with all new inbreeding coefficients, with the exception of $F_{R O H-K S}$ for a minimum number of SNPs greater than 5 .

Figure 4 shows the regression of pedigree-based inbreeding coefficients on $F_{R O H-E}^{p}$ (correlation $=0.70$ ) and on total $\mathrm{ROH}$ content inbreeding coefficients (correlation $=0.68$ ). Some values of $F_{p e d}$ correspond to many values for $F_{R O H-E}^{p}$ and $F_{R O H}$, suggesting that $\mathrm{ROH}$ inbreeding coefficients incorporate segregation within families, in contrast to the pedigree-based coefficients. The three groups of sows (S, C1, and C2) can also be distinguished as clusters of dots in each of the two regressions. The use of the distribution of $\mathrm{ROH}$-length is not restricted to the estimation of the individual inbreeding coefficients but it can also be used to explore differences in inbreeding on different chromosomes. Table 3 provides correlations between chromosomal length and genomic inbreeding coefficients, which show that chromosome length is correlated with $F_{R O H-Q}$ and to a lesser extent with $F_{R O H-E}^{p}$ and $F_{R O H-E}^{m}$, suggesting that these inbreeding coefficients are very much affected by chromosomal length. A very high correlation between $F_{R O H-E}^{p}$ and $F_{R O H-E}^{m}$ was observed, which may be due to the high dependency of these two parameters on the "rate" of the exponential distribution. Figure 5 shows $\mathrm{Q}-\mathrm{Q}$ plots of the distribution of quantiles for each chromosome when plotted against the distribution for all other chromosomes from all individuals. Chromosome 1 had the greatest $F_{R O H-Q}$ values, while

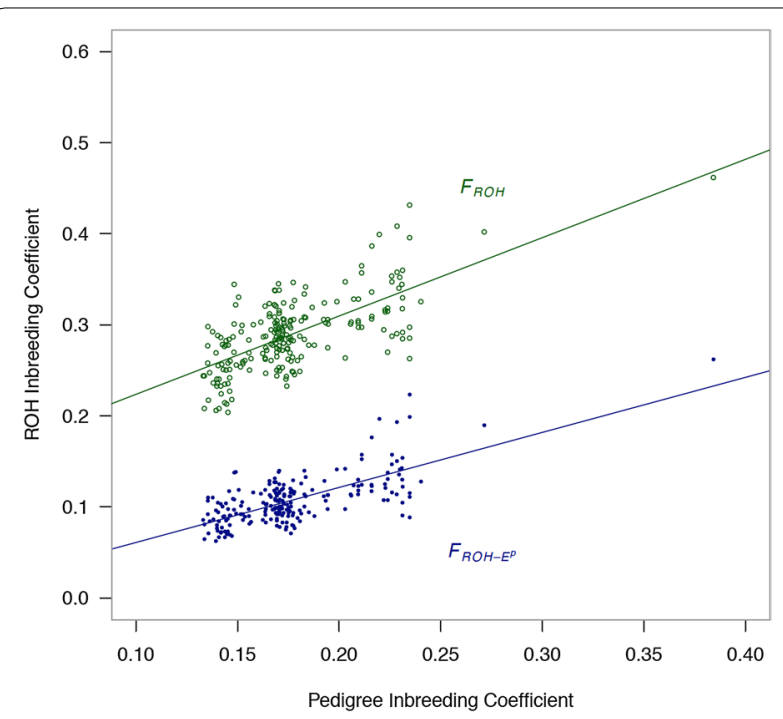

Fig. 4 Regressions of $F_{R O H-E}^{P}$ and $F_{R O H}$ on pedigree inbreeding coefficients $\left(F_{\text {ped }}\right)$. Minimum number of SNPs to declare a $\mathrm{ROH}>5$ for $F_{R O H-E}^{P}$ and $\mathrm{T}=2 \mathrm{Mb}$ and $\mathrm{ROH}$ measured in $\mathrm{Mb}$ chromosome 10 had the lowest $F_{R O H-Q}$ values and $\mathrm{ROH}$ fragments that were shorter than those for the rest of the genome. These results illustrate the relationship between $F_{R O H-Q}$ and chromosomal length.

The use of the distribution of $\mathrm{ROH}$ length to measure inbreeding does not only open new possibilities to investigate autozygosity at the chromosomal level but also to investigate old established principles and knowledge on inbreeding. In 1961, Alan Robertson [27] postulated that inbreeding must increase in populations under selection. Figure 6 shows $\mathrm{Q}-\mathrm{Q}$ plots of the distribution of the length of $\mathrm{ROH}$ for the unselected groups $\mathrm{C} 1$ and $\mathrm{C} 2$ and for the selected group $\mathrm{S}$ relative to the $\mathrm{ROH}$ distribution of all individuals. A clear pattern can be observed, showing that the $\mathrm{S}$ group had systematically larger $\mathrm{ROH}$ fragments, and therefore, more inbreeding than groups $\mathrm{C} 1$ and $\mathrm{C} 2$. Group $\mathrm{C} 2$ had larger $\mathrm{ROH}$ fragments than $\mathrm{C} 1$, which is attributable to an increase in inbreeding during the five generations that passed between $\mathrm{C} 1$ sows and their descendants in $\mathrm{C} 2$. There was also an increase in pedigree inbreeding coefficients in the selected group $\mathrm{S}$ $(0.21)$ relative to $\mathrm{C} 1(0.14)$ and $\mathrm{C} 2(0.17)$. This increase in inbreeding was also evident for $F_{R O H}(0.21$ for $\mathrm{C} 1,0.25$ for $\mathrm{C} 2$, and 0.28 for $\mathrm{S}$ ) or for $F_{\text {Mol }}(0.65$ for $\mathrm{C} 1,0.65$ for $\mathrm{C} 2$, and 0.67 for S). Similarly, exponential inbreeding coefficients were higher for the selected group, S. In summary, selection has impacted the genetic variability of the $S$ group, as detected by both pedigree and molecular-based indicators of inbreeding.

The next question was to investigate whether the increase in length of $\mathrm{ROH}$ was more marked in some chromosomes as a result of selection. Figure 7 shows $\mathrm{Q}-\mathrm{Q}$ plots of the S group versus the non-selected C1 group by chromosome. Most chromosomes had longer $\mathrm{ROH}$ in the $\mathrm{S}$ group. The distributions in both groups were similar for chromosome 4, while the $\mathrm{C} 1$ group had some larger $\mathrm{ROH}$ fragments for chromosome 13. Figure 8 shows the length of each $\mathrm{ROH}$ along their position on chromosomes 5, 9 and 16. These chromosomes were chosen because of the larger sizes of $\mathrm{ROH}$ fragments in the $\mathrm{S}$ group versus $\mathrm{C} 1$ (Fig. 7). There were $\mathrm{ROH}$ of larger sizes at the beginning of chromosomes 5 and 16. Large $\mathrm{ROH}$ fragments were distributed evenly along chromosome 9.

\section{Discussion}

By "genomic inbreeding coefficient", we denote a parameter that uses genomic information on autozygosity as a measure of relatedness among ancestors of an individual. It includes molecular inbreeding coefficients, $\mathrm{ROH}$ inbreeding coefficients $[10-19,28,29]$ and coefficients that make use of the length of $\mathrm{ROH}$ as a random variable with an associated probability distribution or probability density function, as proposed in this paper. One of the 
Table 3 Correlations between pairs of chromosomal genomic inbreeding coefficients and length (number of SNPs per chromosome)

\begin{tabular}{|c|c|c|c|c|c|c|c|}
\hline & Length & $F_{M o l}$ & $F_{\mathrm{ROH}}$ & $F_{\mathrm{ROH}-\mathrm{KS}}$ & $F_{\mathrm{ROH}-\mathrm{Q}}$ & $F_{R O H-E}^{m}$ & $F_{R O H-E}^{p}$ \\
\hline Length & & 0.04 & 0.16 & 0.57 & 0.84 & 0.57 & 0.57 \\
\hline$F_{M O l}$ & 0.25 & & 0.45 & 0.01 & -0.07 & 0.44 & 0.45 \\
\hline$F_{R O H}$ & 0.25 & 0.25 & & 0.32 & 0.13 & 0.51 & 0.52 \\
\hline$F_{R O H-K S}$ & 0.21 & 0.25 & 0.24 & & 0.60 & 0.83 & 0.82 \\
\hline$F_{\mathrm{ROH}-\mathrm{Q}}$ & 0.14 & 0.25 & 0.25 & 0.20 & & 0.59 & 0.58 \\
\hline$F_{R O H-E}^{m}$ & 0.20 & 0.22 & 0.21 & 0.14 & 0.20 & & 0.99 \\
\hline$F_{R O H-E}^{P}$ & 0.21 & 0.22 & 0.21 & 0.14 & 0.20 & 0.00 & \\
\hline
\end{tabular}

Correlations are on the upper diagonal; approximate standard errors are on the lower diagonal; $F_{R O H-K S}, F_{R O H-Q_{Q}} F_{R O H-E}^{m}$ and $F_{R O H-E}^{p}$ were computed for a minimum of five SNPs and measured as physical distance in Mb; quantiles in $F_{R O H-Q}$ were computed excluding the chromosome being tested from the reference population; $F_{R O H-E}^{P}$ were computed for a threshold of $\mathrm{T}=5 \mathrm{Mb}$

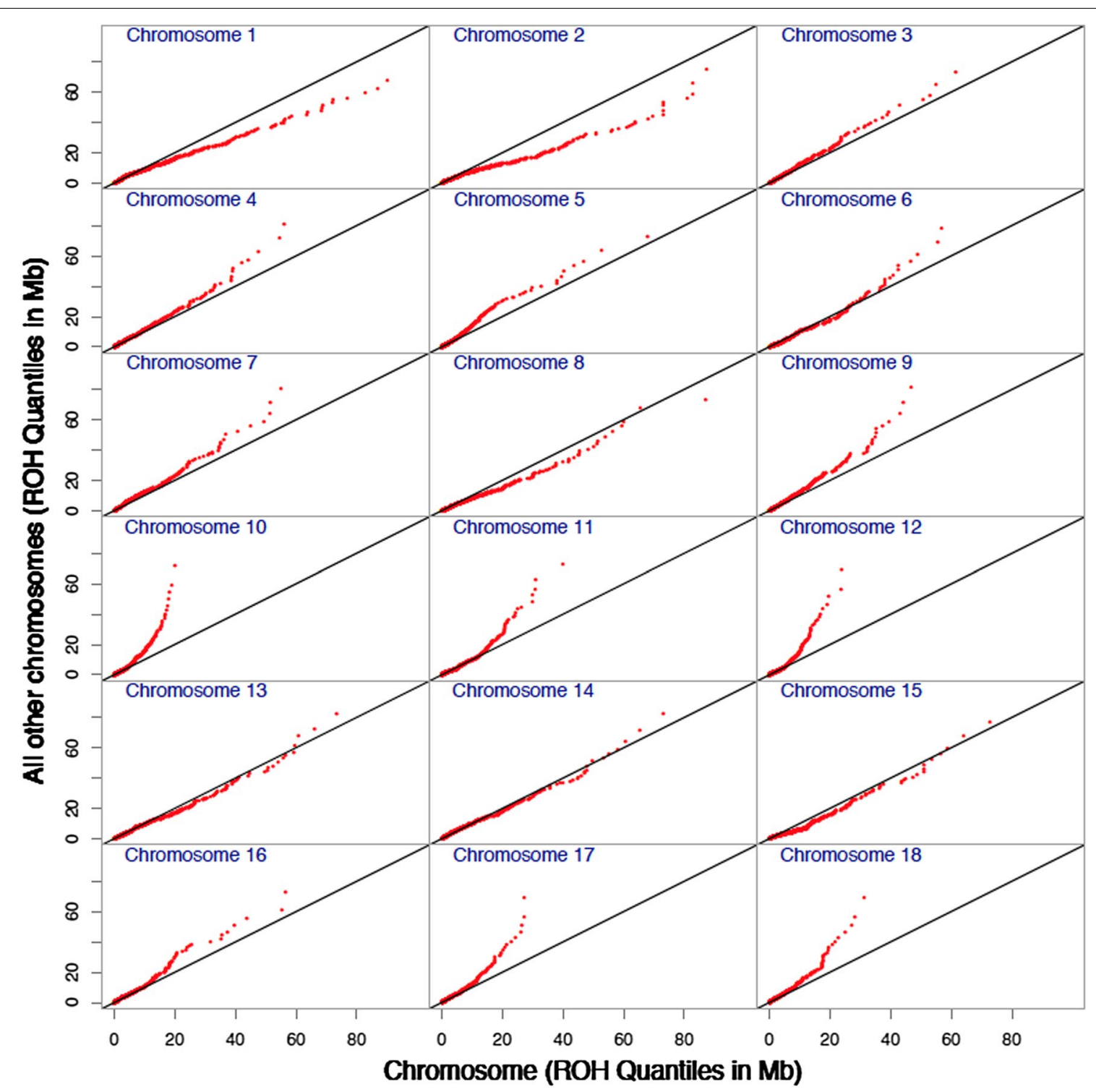

Fig. 5 Q-Q plots of the distribution of the length of ROH for each of the 18 autosomes versus all chromosomes. The chromosome being tested was not included in the reference population; minimum number of SNPs to declare a $\mathrm{ROH}>5, \mathrm{ROH}$ measured in $\mathrm{Mb}$ 


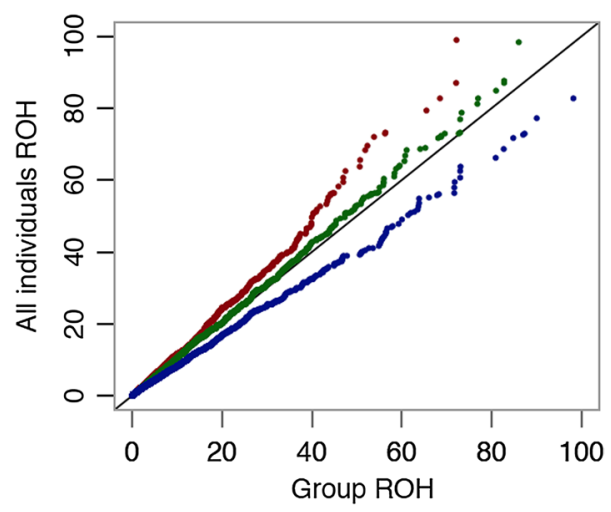

Fig. 6 The effect of selection on the distribution of the length of $\mathrm{ROH}$. Q-Q plots of the distribution of ROH fragment sizes for all individuals versus the distribution for individuals C1 (dark-red), C2 (dark-green), and S (dark-blue); minimum number of SNPs to declare a $\mathrm{ROH}>5, \mathrm{ROH}$ measured in $\mathrm{Mb}$

first issues that had to be addressed is how to estimate ROH. DNA sequencing methods are required to observe autozygosity but often $\mathrm{ROH}$ are estimated based on genotypes obtained with BeadChip arrays of SNPs. Stretches of DNA are declared as $\mathrm{ROH}$ if a minimum number of consecutive SNPs from an array are homozygous. We explored four different minimum numbers of SNPs to declare a $\mathrm{ROH}(5,15,25$, and 35$)$ and considered two alternative measures of length, the number of SNPs and physical length in $\mathrm{Mb}$. Our results suggest that the minimum number of SNPs can affect correlations between genomic and pedigree inbreeding coefficients. On the one hand, Quantile and Kolmolgorov-Smirnov $\mathrm{ROH}$ inbreeding coefficients were less correlated with pedigree inbreeding coefficients when the minimum number was small, in contrast to exponential inbreeding coefficients. Nevertheless, differences between inbreeding coefficients based on ROH length were not large, except for $F_{R O H-K S}$. On the other hand, correlations between pedigree and genomic inbreeding coefficients were slightly higher when using the $\mathrm{ROH}$ length based on number of SNPs instead of physical distance. An explanation is that only some SNPs in a DNA fragment are genotyped and errors in declaring a fragment autozygous add another source of error to the usual genotyping errors, such as SNP location or distance between SNPs in the array. Nevertheless, the correlations based either on the number of SNPs or on physical distance were rather similar across all situations investigated.

All inbreeding coefficients (traditional and newly developed) have advantages and disadvantages. The advantage of the pedigree inbreeding coefficient is that it is simple and only requires recording of pedigrees but it does not account for the sampling that occurs when gametes are produced during meiosis. That is, pedigree inbreeding coefficients are probabilistic and do not account for the fact that individuals with the same inbreeding history can differ in autozygosity. For example, two full-sibs can have different numbers of fragments of autozygosity (and at different locations) just by sampling. In contrast, all genomic inbreeding coefficients account for sampling and they measure the "realized inbreeding" of an individual.

Genomic inbreeding coefficients differ in the way they use the genotype information. Molecular inbreeding coefficients are calculated as the proportion of homozygous sites that are genotyped with an array. They assume that the genotyped SNPs are randomly located across the genome and do not distinguish old from recent inbreeding. This coefficient incorporates the entire breeding history of the individual, including new mutations and old inbreeding. The total $\mathrm{ROH}$ content inbreeding coefficient is the proportion of the genome of an individual that comprises autozygous fragments. This coefficient does incorporate regions of autozygosity but, in contrast to the molecular coefficient, it ignores fragments consisting of a single or a few contiguous homozygous SNPs in its computation. Total $\mathrm{ROH}$ content inbreeding coefficient does distinguish old from recent inbreeding but with the limitation that direct information on the length of $\mathrm{ROH}$ fragments is not used. In principle, two individuals with the same total $\mathrm{ROH}$ content inbreeding coefficients can have a different proportion of large and short $\mathrm{ROH}$ fragments. However, total $\mathrm{ROH}$ content inbreeding coefficients may indirectly account for the length of $\mathrm{ROH}$ because highly inbred animals, such as progeny from the mating between two full-sibs, should have larger total $\mathrm{ROH}$ content inbreeding coefficients made up by a large number of $\mathrm{ROH}$ of larger size.

The inbreeding coefficients proposed in this paper do incorporate direct information on the length of $\mathrm{ROH}$ to a greater or lesser extent. The Kolmogorov-Smirnov coefficient is a general method to compare statistical distributions and was used here to discern individuals with a very different distribution of $\mathrm{ROH}$ length when compared to the rest of the population. The Quantile inbreeding coefficient is very well suited to detect individuals with larger $\mathrm{ROH}$ fragments due to recent inbreeding and it leads to a graphical representation of the inbreeding of an individual. The exponential mean and variance inbreeding coefficients assume that one single parameter, the rate of the exponential distribution, defines the inbreeding status of an individual. A higher rate means that the individual has a greater average length of $\mathrm{ROH}$ fragments. For simplicity, the reference population was a pool of all $\mathrm{ROH}$ fragments of all individuals. This part corresponding to the reference population could be better represented by 


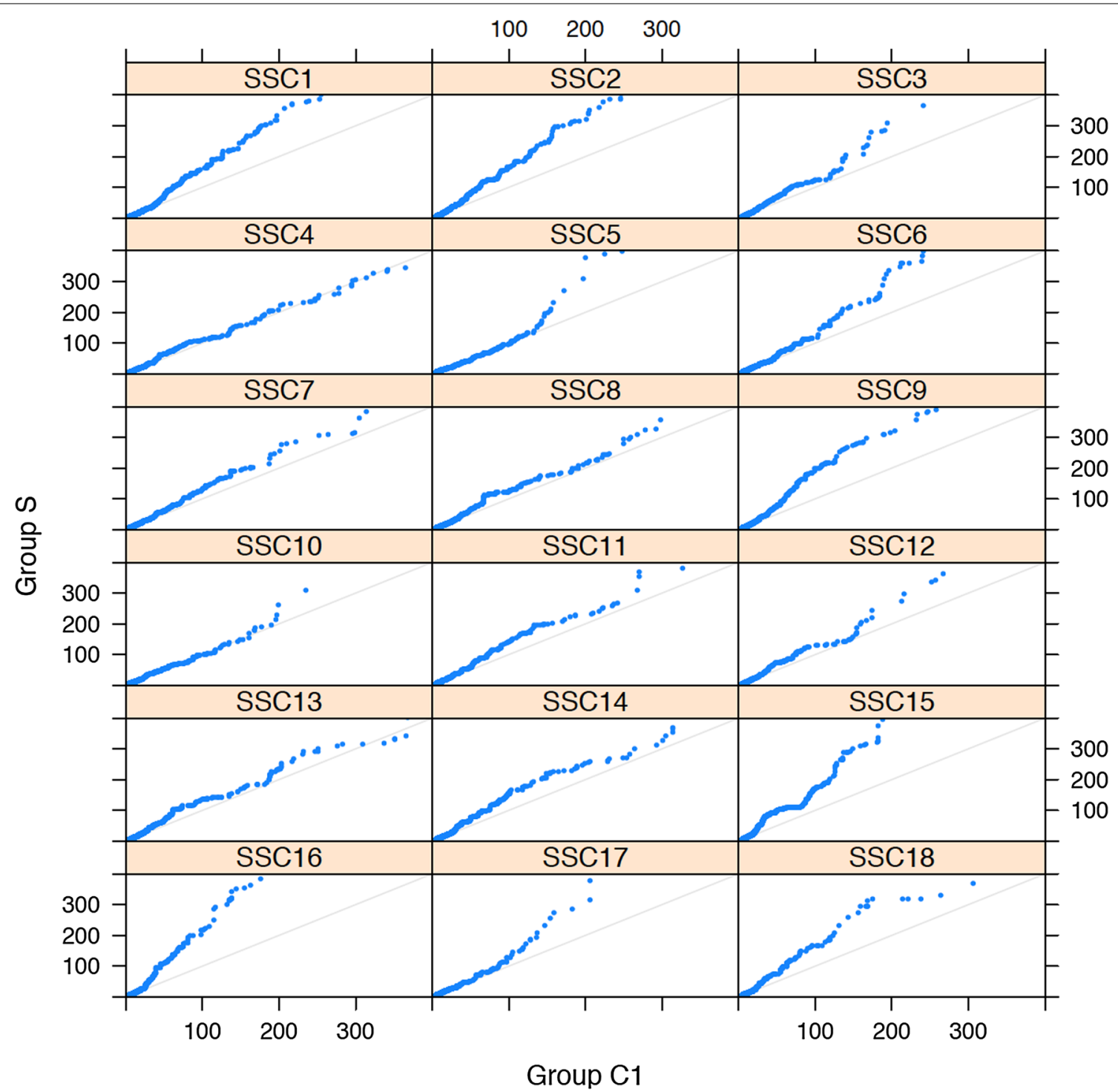

Fig. $7 \mathrm{Q}-\mathrm{Q}$ plots of the distribution of ROH for selected group S versus group C1 for each of the 18 autosomes. Minimum number of SNPs to declare a $\mathrm{ROH}>5$, $\mathrm{ROH}$-length measured by the number of contiguous homozygous SNPs

fitting a gamma distribution instead of an exponential distribution since the sum of exponentials follows this distribution. Kolmogorov-Smirnov, Quantile, and exponential inbreeding coefficient do not fall within the range of $0-1$ (in contrast to the pedigree, molecular, and total $\mathrm{ROH}$ content inbreeding coefficients) but they could easily be standardized (i.e., forced to be between 0 and 1 ) by:

\section{Standardized inbreeding of the ith individual}

$$
=\frac{F_{i}-\min \left(F_{R O H}\right)}{\max \left(F_{R O H}\right)-\min \left(F_{R O H}\right)}
$$

where $F_{i}$ is the inbreeding coefficient of the $i$-th individual before it is standardized and $F_{R O H}$ is its distribution
(K-S, quantile or exponential). However, the standardized inbreeding coefficients do not abide by the definition of an inbreeding coefficient (i.e., the probability that two alleles at a locus in an individual are identical by descent), and cannot be used to compare individuals from populations with a different inbreeding history. The exponential $p$ inbreeding coefficient does range from 0 to 1 , since it is defined as a probability. The exponential- $p$ inbreeding coefficient requires definition of a threshold, $\mathrm{T}$, which should be the same when comparing inbreeding of different individuals from the same population. More work is needed to explore the impact of alternative thresholds on estimates of inbreeding coefficients of animals from different populations. 


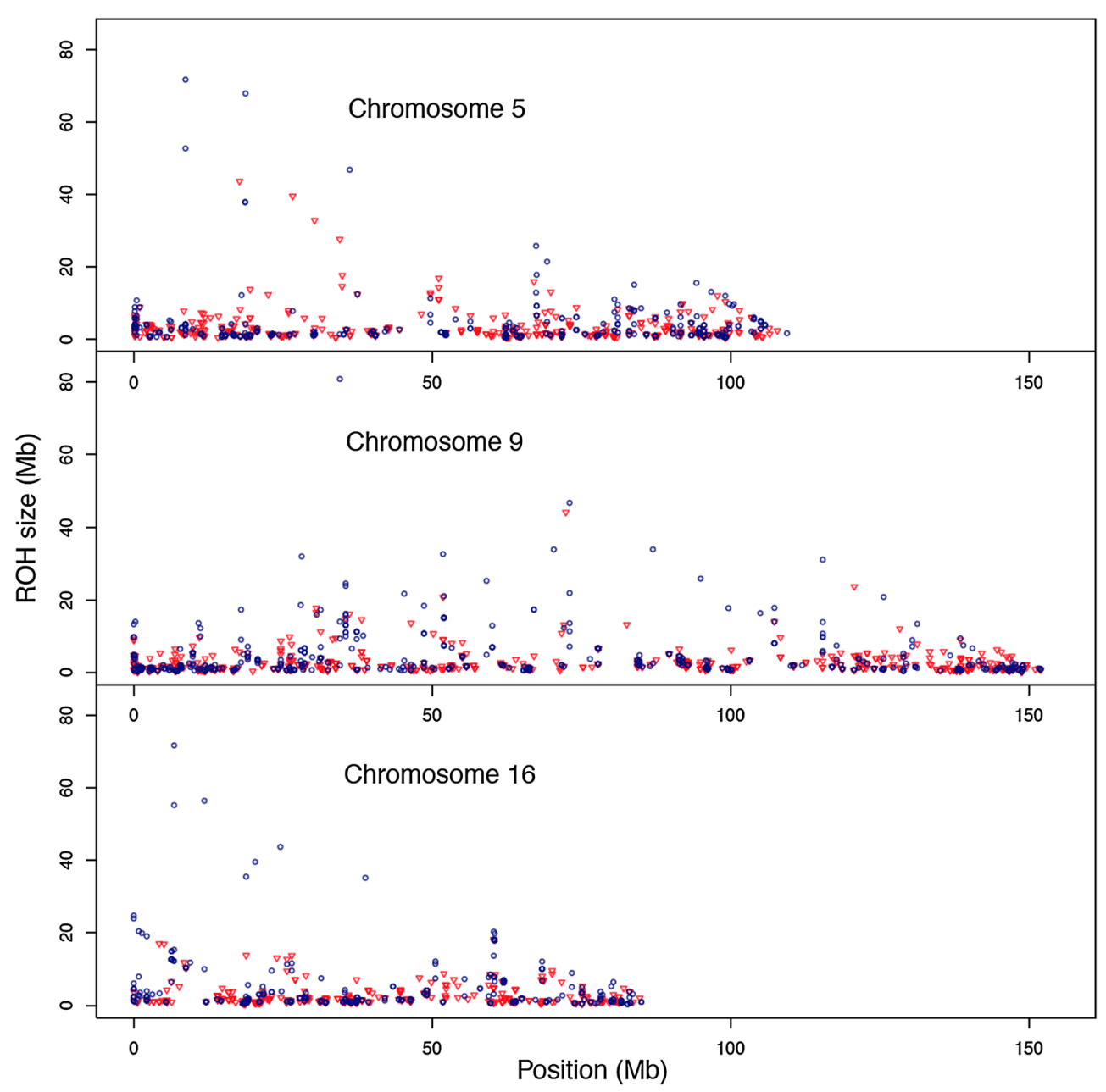

Fig. 8 Distribution of $\mathrm{ROH}$-length along chromosomal positions. The figure represents conservation group ( $\mathrm{C} 1$ color red), and selected group (S color navy) on chromosomes 5, 9, and 16 for a minimum number of SNPs to declare a ROH >15 and measured in Mb

Comparison of the new metrics to existing methods provides little information on their ability to detect long $\mathrm{ROH}$ (as an aid to detect recent inbreeding) since existing methods cannot. Thus, in order to investigate the ability of the new methods to detect long $\mathrm{ROH}$ fragments, correlations between chromosomal inbreeding and chromosomal length were performed. A recent common ancestor of the parents of an individual is expected to result in entire chromosomes or long DNA fragments (as a result of single or multiple recombination events in the different paths leading to the parents of the individual) to be identical by descent in the individual. Therefore, long chromosomes are expected to result in longer $\mathrm{ROH}$ fragments. In addition, longer chromosomes have been shown to have a lower recombination rate $(\mathrm{cM} / \mathrm{Mb})$ in swine [30], which would also result in longer $\mathrm{ROH}$ fragments. Our results show that chromosomal length was highly correlated with quantile chromosomal inbreeding coefficients and to a lesser extent with other proposed metrics. Thus, quantile inbreeding coefficients are sensitive to long $\mathrm{ROH}$ fragments and, therefore, improve detection of recent inbreeding.

The largest limitation of the newly proposed metrics is that they do not allow for straightforward comparison of the level of inbreeding of individuals from different species. Genomes with different number and size of chromosomes (or recombination rate) may lead to distributions of individual inbreeding coefficients based on $\mathrm{ROH}$ length that are not comparable. This may be overcome by using exponential- $p$ inbreeding coefficients and by setting appropriate thresholds that facilitate comparisons across species. For example, 
thresholds could be chosen based on the distributions of $\mathrm{ROH}$ length for each species relative to the distribution of $\mathrm{ROH}$ length of several species together.

Traditional and new inbreeding coefficients allowed for the detection of the effect of selection on inbreeding [27]. However, the genomic inbreeding coefficients can pinpoint chromosomal regions where autozygosity is more extensive. Selection has two effects on inbreeding: one is its direct action to increase the frequency of alleles that favorably affect the trait under selection; the other is the increase in inbreeding and autozygosity for all loci regardless of their effects on the trait, which is attributed to co-selection of individuals with high breeding values which tend to share not just alleles at loci with an effect on the trait but at all loci, i.e., to be relatives [31]. Our results for chromosomes 5, 9 and 16 support the hypothesis that autozygosity affects both loci that are related to the selected traits as well as neutral loci scattered over the genome. In addition, the increased autozygosity in the $\mathrm{S}$ group is apparent for all chromosomes except chromosome 4. The method can identify chromosomal inbreeding but not the reasons for its occurrence.

In conservation genetics, coancestry coefficients are used to optimize genetic management in a conservation program and several estimators of coancestries based on molecular information have been proposed, e.g., [32, 33]. These methods ignore that linked SNPs are inherited together, and consequently, the information provided by ROH. Pryce et al. [34] showed that $\mathrm{ROH}$ could provide additional information on coancestry when mating relatives. However, their approach consisted in estimating the proportion of haplotypes at a given length of $\mathrm{ROH}$ that are common between individuals. A novel alternative would be to make use of the expected distribution of the length of the $\mathrm{ROH}$ among progeny of related parents, in line with our proposed use of $\mathrm{ROH}$ to quantify inbreeding. In other words, to use coancestry coefficients based on the expected shape of the distribution of $\mathrm{ROH}$ lengths in the progeny of the two parents.

\section{Conclusions}

The proposed inbreeding coefficients add to existing methods to estimate inbreeding by accounting for the length of $\mathrm{ROH}$, which incorporates information on recent inbreeding. Among the proposed metrics, quantile inbreeding coefficients are the most sensitive for identifying individuals with longer $\mathrm{ROH}$ fragments. Exponential$p$ inbreeding coefficients are less sensitive for detecting long $\mathrm{ROH}$ fragments but are defined as a probability (they range from 0 to 1 ) and are, therefore, suitable for comparison of individuals across populations.

\section{Additional files}

Additional file 1. Supplemental.Running.example.June2015.docx. This file contains instructions for running an example for the new inbreeding coefficients.

Additional file 2. Inbreeding.example. This file contains a small dataset with information on $\mathrm{ROH}$ length for two individuals on chromosome 1. The variables are: nchro: number of chromosome; ind: number of individuals; gr: group; po1: initial chromosomal position; po2: final chromosomal position; roh: $\mathrm{ROH}$ length in number of homozygous SNPs in the segment.

Additional file 3. $k$-s.r. Source code in R for estimating KolmogorovSmirnov inbreeding coefficients.

Additional file 4. Quantile.r. Source code in R for estimating quantile inbreeding coefficients.

Additional file 5. Expon.r. Source code in $\mathrm{R}$ for estimating exponential inbreeding coefficients.

\section{Authors' contributions}

$L S$ and CR conceived and carried out the genotyping experiments, helped in the data analysis, and contributed greatly with the writing of the manuscript; LGR conceived the idea of using the fragments of $\mathrm{ROH}$ as a random variable, analyzed the data and wrote the first version of the manuscript; CB selected the genotyped samples, edited the raw SNP data and helped to write the manuscript. All authors read and approved the final manuscript.

\section{Acknowledgements}

The authors are grateful to Wendy M. Rauw for criticism of the manuscript. Technical assistance of Fabián García is gratefully acknowledged. Financial support was provided by RTA2011-00113 and RZ2012-00006 grants. We acknowledge the effort of Jaime Rodrigáñez and all the staff of the Iberian pig farm 'Dehesón del Encinar' for maintaining strict pedigree and data recording on the Torbiscal pigs and their ancestors since 1944 until the recent closure of the farm facilities. The authors are thankful to two anonymous reviewers for their comments to improve the manuscript.

\section{Consent for publication}

The cover image belongs to the author and therefore no consent is required.

\section{Competing interests}

The authors declare that they have no competing interests.

Received: 28 November 2014 Accepted: 13 September 2015

Published online: 16 October 2015

\section{References}

1. Crow JF, Kimura M. An introduction to population genetics theory. New York: Harper and Row; 1970.

2. Ouborg NJ, Pertoldi C, Loeschcke V, Bijlsma R, Hedrick PW. Conservation genetics in transition to conservation genomics. Trends Genet. 2010;26:177-87

3. Leroy G. Inbreeding depression in livestock species: review and metaanalysis. Anim Genet. 2014;45:618-28.

4. Keller LF, Waller DM. Inbreeding effects in wild populations. Trends Ecol Evol. 2002;17:230-41.

5. Wright S. Coefficients of inbreeding and relationship. Am Nat. 1922;56:330-8.

6. Toro MA, Fernández J, Caballero A. Molecular characterization of breeds and its use in conservation. Livest Sci. 2009;120:174-95.

7. VanRaden PM. Efficient methods to compute genomic predictions. J Dairy Sci. 2008;91:4414-23.

8. Toro MA, García-Cortés LA, Legarra A. A note on the rationale for estimating genealogical coancestry from molecular markers. Genet Sel Evol. 2011;43:27. 
9. Gibson J, Morton NE, Collins A. Extended tracts of homozygosity in outbred human populations. Hum Mol Genet. 2006;15:789-95.

10. Keller MC, Visscher PM, Goddard ME. Quantification of inbreeding due to distant ancestors and its detection using dense single nucleotide polymorphism data. Genetics. 2011;189:237-49.

11. McQuillan R, Leutenegger AL, Abdel-Rahman R, Franklin CS, Pericic M, Barac-Lauc L, et al. Runs of homozygosity in European populations. Am J Hum Genet. 2008;83:359-72.

12. Nothnagel M, Lu TT, Kayser M, Krawczak M. Genomic and geographic distribution of SNP-defined runs of homozygosity in Europeans. Hum Mol Genet. 2010;19:2927-35.

13. Kirin M, McQuillan R, Franklin CS, Campbell H, McKeigue PM, Wilson JF. Genomic runs of homozygosity record population history and consanguinity. PLoS One. 2010;5:e13996.

14. Ferenčaković M, Hamzic E, Gredler B, Curik I, Solkner J. Runs of homozygosity reveal genome-wide autozygosity in the Austrian fleckvieh cattle. Agric Conspec Sci. 2011;76:325-9.

15. Pryce JE, Haile-Mariam M, Goddard ME, Hayes BJ. Identification of genomic regions associated with inbreeding depression in Holstein and Jersey dairy cattle. Genet Sel Evol. 2014;46:71.

16. Bosse M, Megens HJ, Madsen $O$, Paudel Y, Frantz LAF, Schook LB, et al. Regions of homozygosity in the porcine genome: consequence of demography and the recombination landscape. PLoS Genet. 2012;8:e1003100.

17. Silió L, Rodríguez MC, Fernández A, Barragán C, Benítez R, Ovilo C, et al. Measuring inbreeding and inbreeding depression on pig growth from pedigree or SNP-derived metrics. J Anim Breed Genet. 2013;130:349-60.

18. Leutenegger AL, Prum B, Génin E, Verny C, Lemainque A, Clerget-Darpoux $F$, et al. Estimation of the inbreeding coefficient through use of genomic data. Am J Hum Genet. 2003;73:516-23.

19. Ferenčaković M, Hamzić E, Gredler B, Solberg TR, Klemetsdal G, Curik I, et al. Estimates of autozygosity derived from runs of homozygosity: empirical evidence from selected cattle populations. J Anim Breed Genet. 2013;130:286-93.

20. Fernandez A, Rodrigáñez J, Toro MA, Rodrıguez MC, Silió L. Inbreeding effects on the parameters of the growth function in three strains of Iberian pigs. J Anim Sci. 2002;80:2267-75.

21. Woolliams JA, Mäntysaari EA. Genetic contributions of Finnish Ayrshire bulls over four generations. Anim Sci. 1995;61:177-87.

22. Emik LO, Terrill CE. Systematic procedures for calculating inbreeding coefficients. J Hered. 1949;40:51-55. http://jhered.oxfordjournals.org/ content/40/2/51.extract.
23. Hinrichs D, Meuwissen THE, Ødegard J, Holt M, Vangen O, Woolliams JA. Analysis of inbreeding depression in the first litter size of mice in a longterm selection experiment with respect to the age of the inbreeding. Heredity (Edinb). 2007;99:81-8.

24. Rodríguez C, Núñez Y, Fernández Al, Fernández A, Barragán C, Silió L. Tracking footprints of an experiment of selection in Iberian pigs. In: Proceedings of the 10th World Congress of Genetics Applied to Livestock Production: 17-22 August 2014; Vancouver; 2014. https://asas.org/docs/ default-source/wcgalp-posters/934_paper_9335_manuscript_636_0. pdf?sfursn $=2$.

25. Ramos AM, Crooijmans RP, Affara NA, Amaral AJ, Archibald AL, Beever $J E$, et al. Design of a high density SNP genotyping assay in the pig using SNPs identified and characterized by next generation sequencing technology. PLoS One. 2009;4:e6524.

26. Clark AG. The size distribution of homozygous segments in the human genome. Am J Hum Genet. 1999;65:1489-92.

27. Robertson A. Inbreeding in artificial selection programmes. Genet Res. 1961;2:189-94

28. Howrigan DP, Simonson MA, Keller MC. Detecting autozygosity through runs of homozygosity: a comparison of three autozygosity detection algorithms. BMC Genom. 2011;12:460.

29. Pemberton TJ, Asher D, Feldman MW, Myers RM, Rosenberg NA, Li JZ. Genomic patterns of homozygosity in worldwide Human populations. Am J Hum Genet. 2012;91:275-92.

30. Muñoz M, Alves E, Ramayo-Caldas Y, Casellas J, Rodriguez C, Folch JM, et al. Recombination rates across porcine autosomes inferred from highdensity linkage maps. Anim Genet. 2012;43:620-3.

31. Belonsky GM, Kennedy BW. Selection on individual phenotype and best linear unbiased predictor of breeding value in a closed swine herd. J Anim Sci. 1998;66:1124-31.

32. Toro M, Barragán C, Ovilo C, Rodrigáñez J, Rodriguez C, Silió L. Estimation of coancestry in Iberian pigs using molecular markers. Conserv Genet. 2002;3:309-20.

33. Oliehoek PA, Windig JJ, van Arendonk JAM, Bijma P. Estimating relatedness between individuals in general populations with a focus on their use in conservation programs. Genetics. 2006;173:483-96.

34. Pryce JE, Hayes BJ, Goddard ME. Novel strategies to minimize progeny inbreeding while maximizing genetic gain using genomic information. J Dairy Sci. 2012;95:377-88.

\section{Submit your next manuscript to BioMed Central and take full advantage of:}

- Convenient online submission

- Thorough peer review

- No space constraints or color figure charges

- Immediate publication on acceptance

- Inclusion in PubMed, CAS, Scopus and Google Scholar

- Research which is freely available for redistribution

Submit your manuscript at

www.biomedcentral.com/submit

C BioMed Central 